\title{
Two Novel Forms of ERG Oscillation in Drosophila: Age and Activity Dependence
}

\author{
Atsushi Ueda*, Scott Woods*, Ian McElree*, Tristan C. D. G. O’Harrow, Casey Inman ${ }^{+}$, \\ Savantha Thenuwara, Muhammad Aftab, Atulya Iyengar ${ }^{\#}$ \\ Department of Biology, University of Iowa, Iowa City, IA 52242, USA.

\section{Running Title: Novel ERG oscillations in Drosophila}

Key Words: On-Transient, Off-Transient, Receptor Potential, Countercurrent Apparatus, Aging, Lifespan

* These authors contributed equally.

${ }^{+}$Present address: Washington University School of Medicine, St. Louis, MO 63110

${ }^{\#}$ Corresponding author:

Dr. Atulya Iyengar

Department of Biology

University of Iowa

Iowa City, IA 52242

(319) 335-1090

atulya-iyengar@uiowa.edu

\section{Manuscript Information:}

Number of Pages: 20,, Number of Figures: 5, Number of Tables: 1 Number of Supplemental Figure: 1.

\section{Acknowledgments}

We thank Prof. Chun-Fang Wu for supervising the Neurobiology Laboratory course and for providing his support and encouragement to develop this work. We also thank Prof. Bernd Fritzsch, Prof. Michael Dailey and Dr. Olga Miakotina for organizational and technical assistance. We thank other participants of the course for their assistance in data collection. AU and AI were supported by the NIH grant AG051513 to Chun-Fang Wu. 


\section{Abstract}

Over an animal's lifespan, neuronal circuits and systems often decline in an inherently heterogeneous fashion. To compare the age-dependent progression of changes in visual behavior with alterations in retinal physiology, we examined phototaxis and electroretinograms (ERGs) in a wild-type D. melanogaster strain $($ Canton-S) across their lifespan. In aged flies (beyond 50\% median lifespan), we found a marked decline in phototaxis, while motor coordination was less disrupted, as indicated by relatively stronger negative geotaxis. These aged flies displayed substantially reduced ERG transient amplitudes while the receptor potentials (RP) remained

9 largely intact. Using a repetitive light flash protocol, we serendipitously discovered two forms of activity-dependent oscillation in the ERG waveforms of young flies: "light-off" and "light-on" oscillations. After repeated $500 \mathrm{~ms}$ light flashes, light-off oscillations appeared during the ERG off-transients (frequency: 50-120 Hz, amplitude: 1 mV). Light-on oscillations (100-200 Hz,

$13 \sim 0.3 \mathrm{mV}$ ) were induced by a series of $50 \mathrm{~ms}$ flashes, and were evident during the ERG ontransients. Both forms of oscillation were observed in other strains of D. melanogaster (Oregon$R$, Berlin), additional Drosophila species (funerbris, euronotus, hydei, americana), and were

16 evoked by a variety of light sources. Both light-off and light-on oscillations were distinct from

17 previously described ERG oscillations in visual mutants, such as rosA, in terms of location

18 within the waveform and frequency. However, within $\operatorname{ros} A$ mutants, light-off oscillations, but not

19 light-on oscillations could be recruited by the repetitive light flash protocol. Importantly though,

20 we found that both forms of oscillation were rarely observed in aged flies. Although the

21 physiological bases of these oscillations remain to be elucidated, they may provide important

22 clues to age-related changes in neuronal excitability and synaptic transmission. 


\section{Introduction}

The Drosophila electroretinogram (ERG) provides an accessible and incisive readout of visual system function in a genetically tractable model organism (Pak, 1975; Stark \& Wasserman, 1974; Vilinsky \& Johnson, 2012). The complex waveform represents an extracellular combination of electrical activities associated with phototransduction and synaptic transmission. Prominent features of the ERG waveform include a sustained receptor potential (RP), as well as light on- and light off-transients, with each feature corresponding to a distinct set of underlying physiological processes. The RP component represents the sustained depolarization in photoreceptor cells (Heisenberg, 1971; Alawi \& Pak, 1971), while the transients generally reflect synaptic potentials of the photoreceptor cells and their targets, large monopolar neurons in the optic lamina (Heisenberg, 1971; Coombe, 1986). Recent studies on neurotransmitter re-uptake by adjacent glia have also implicated these cells in shaping the ERG waveform (Rahman, et al., 2012; Chaturvedi, Reddig, \& Li, 2014). Thus, features of the Drosophila ERG waveform serve as a report on the performance of photoreceptors, $2^{\text {nd }}$ order neurons, and surrounding glia in initial visual information processing.

Mutant lines with defective ERG waveforms have provided significant insight into the functions of a number of genes which encode components of $2^{\text {nd }}$ messenger systems, ion channels, and synaptic transmission machinery (Pak, 1975; Pak, 2010). Notable examples include mutants of no receptor potential A (norpA), encoding Phospholipase C (Bloomquist et al., 1988); and transient receptor potential (trp, Minke, Wu, \& Pak, 1975), encoding the founding member of the $\mathrm{Na}^{+} / \mathrm{Ca}^{2+}$-permeable TRP superfamily of cation channels (Montell \& Rubin, 1989; Clapham, Runnels, \& Strubing, 2001). With the advent of transgenic techniques, Drosophila ERGs have proven themselves invaluable by providing first-order assessments of 
neuronal dysfunction due to disease-associated mutations (Yamamoto et al., 2014; Oortveld et al., 2013). Indeed, overexpression of mutant proteins including $\alpha$-Synuclein, (Chouhan et al., 2016), Parkin (West, Elliott, \& Wade, 2015), and Huntingtin (Lee, Yoshihara, \& Littleton, 2003) often leads to age-dependent disruptions of synaptic transmission and cellular morphology, evident in altered ERG waveforms. Despite the widespread use of the ERG in studying the genetics of age-related neurodegeneration, the progression of alterations that the waveform undergoes during normal healthy aging remains to be fully characterized.

As part of the course Neurobiology Laboratory at the University of Iowa, we performed ERGs on wild-type (WT) and several mutant Drosophila strains across their lifespan. Groups of two or three students each designed a series of experiments to assess the impact of genetic perturbations on age-related changes in visual behavior and physiology against WT and relevant controls. We utilized Benzer's countercurrent apparatus (Benzer, 1967) to assess phototaxis, and we performed ERGs to uncover changes in retinal physiology. The technical simplicity of ERG recordings coupled with the physiological insight they provide make the approach ideal for introducing basic concepts of electrophysiology. Due to time constraints and limited sample sizes, individual student groups were oftentimes not able to develop robust conclusions based on their data alone. However, by pooling results from common genotypes across student groups and by conducting a series of follow-up experiments, we were able to compare the timing of agerelated changes in visual behavior with changes in ERG properties. Furthermore, using a repetitive light stimulus protocol consisting of trains of long-duration $(500 \mathrm{~ms})$ or short-duration (50 ms) flashes with varying inter-flash intervals $(0.1-2.5 \mathrm{~s})$, we discovered two novel forms of oscillation in the Drosophila ERG signal. Together, our results highlight the potential for discovering novel age-dependent phenomena in the Drosophila ERG waveform. 


\section{Materials and methods}

\section{Drosophila husbandry}

Flies were collected 0-3 days post-eclosion, and were housed in standard vials containing cornmeal-agar medium (Frankel \& Brosseau, 1968), replaced at least once a week. To accelerate age-related changes in physiology, the initial cohorts of WT flies were reared within a $29{ }^{\circ} \mathrm{C}$ incubator, under constant darkness (apart from door openings and during transferring). All other flies were reared at $23{ }^{\circ} \mathrm{C}$ under a $12: 12 \mathrm{hr}$ light-dark conditions. The WT strain used for longevity experiments was Canton-S (Ruan \& Wu, 2008). Other strains used include the wildtype strains Oregon-R, and Berlin; as well as the mutant strains $\operatorname{ros} A$ (ros $A^{p 213}$; Burg, Geng, Guan, Koliantz \& Pak 1996). The populations of Drosophila species, D. funebris, D. euronotus and D. americana, were gifts from Dr. Bryant McAllister. The D. hydei strain was acquired from Dr. Chun-Fang Wu's stock collection. All strains used had wild-type eye pigmentation.

\section{Phototaxis \& negative geotaxis}

The countercurrent apparatus used for negative geotaxis and phototaxis assays was originally developed by Seymour Benzer (1967). A dim red light in the room facilitated machine operation. Between 7 and 40 flies were loaded into the starting tube of a four-tube countercurrent machine. For phototaxis assays, the apparatus was placed horizontally in a light box, with an LED strip light (SuperNight 5050 LEDs, Ebestrade, Portland, OR) placed approximately $2 \mathrm{~cm}$ from the machine. For negative geotaxis assays, the apparatus was positioned vertically. To start each round of taxis, the machine was "banged down" to settle the flies. The flies were allowed to move to the opposite tube for $20 \mathrm{~s}$, after which the opposite tube was advanced to the next tube of the apparatus. After three rounds, the number of flies able to successfully traverse to the 
1 opposite tube zero, one, two or three times was recorded. The phototaxis and negative geotaxis indices were computed by finding the average number of tubes traversed by flies of a given age.

\section{ERG recordings}

ERG recording procedures have been described previously (Pak, Grossfield, \& White, 1969; Dolph, Nair, \& Raghu, 2010). Anesthetized flies were mounted into the holes of a breadboard to restrain movement and expose the head. Melted polyethylene glycol wax (melting point: $45-50{ }^{\circ} \mathrm{C}$ ) was applied to the back of the head to secure the fly to the breadboard. Initial experiments utilized ethyl ether as an anesthetic while later experiments used $\mathrm{CO}_{2}$. We did not detect any differences in ERG waveforms based on which anesthetic was used. Flies were

11 allowed to recover at least 15 min prior to ERG recordings. ID, WPI, Sarasota, FL) using an electrode-puller (Model PP-83, Narishige Scientific Instrument Lab, Tokyo, Japan). Electrodes were filled with a saline solution $(0.7 \% \mathrm{w} / \mathrm{v} \mathrm{NaCl})$ and inserted into an electrode holder containing a chloridized silver wire. The electrode resistance was $\sim 1$ $\mathrm{M} \Omega$. The recording electrode was inserted into the cornea at an approximately normal angle. The ground electrode tip was broken and inserted into the proboscis. Signals were amplified 10x by a DC amplifier (IX1, Dagan Corporation, Minneapolis, USA). The amplified signal was sampled LED driven at constant voltage. The LED was positioned towards the eye, approximately $4 \mathrm{~cm}$ away, and the light intensity at the eye was 1,200 lux. Every fly examined underwent six light 
1 flash trains, each train consisting of $60 \mathrm{~s}$ of dark adaptation followed by ten light flashes. Light

2 flashes during each train were either 50 or $500 \mathrm{~ms}$ long, and had an inter-flash interval of $0.1,0.5$

3 or $2.5 \mathrm{~s}$.

$4 \quad$ Data analysis

6 MA) to quantify on-transients, off-transients, receptor potentials and other notable features. 


\section{Results}

\section{Age-related changes in phototaxis and geotaxis}

Progressive decline in behavioral performance is commonly associated with aging. Using Benzer's countercurrent apparatus (1967), we examined the phototactic responses of two populations of WT (Canton-S) flies with distinct lifespan curves: A high temperature-reared group $\left(29^{\circ} \mathrm{C}\right.$ ) with a compressed lifespan (median $\sim 30 \mathrm{~d}$ ), and a room temperature-reared group $\left(23{ }^{\circ} \mathrm{C}\right.$ ) with a relatively longer lifespan (median $\sim 65 \mathrm{~d}$ ). At both rearing temperatures, we observed initially strong phototactic response in young flies followed by a progressive decline in phototactic behavior across the lifespan (Figure 1). Indeed, beyond 50\% of the median lifespan for the two rearing temperatures ( $15 \mathrm{~d}$ and $32 \mathrm{~d}$ respectively), the phototaxis index was substantially reduced compared to younger counterparts. To determine if the observed decline in phototactic response was due to a corresponding loss of motor coordination, we subjected the same populations of flies to a negative geotaxis assay using the same countercurrent apparatus (see Methods). Importantly, across ages displaying marked decline in phototaxis, the negative geotaxis indices were consistently higher than corresponding phototaxis indices. This difference was most prominent at $18 \mathrm{~d}$ for $29{ }^{\circ} \mathrm{C}$-reared flies $(1.4 \mathrm{vs} 2.2$, Figure $1 \mathrm{~A})$ and at $30 \mathrm{~d}$ for $23{ }^{\circ} \mathrm{C}$ reared flies (0.4 vs 2.0, Figure 1B). Our findings suggests that age-related decline in phototaxis precedes general loss of motor coordination, and may be due to alterations in visual system function.

\section{Age-related changes in ERG}

To correlate the observed decline in visual system function with changes in retinal physiology, we recorded ERGs from $29^{\circ} \mathrm{C}$-reared WT flies across their lifespan (Figure 2). In young flies responding to a $500 \mathrm{~ms}$ light flash, we observed robust receptor potentials (RP, $~ 10$ 
$1 \mathrm{mV})$ as well as on-transients $(\sim 2 \mathrm{mV})$ and off-transients $(\sim 5 \mathrm{mV}$, Figure $2 \mathrm{~A})$. Across all ages examined, we found the RP amplitude was largely maintained (Figure 2B). However, as flies aged, we observed a substantial reduction in the amplitudes of on- and off-transients (Figure 2A). Indeed, in most flies older than $15 \mathrm{~d}$, the on-transients were undetectable (5/8 flies, Figure $2 \mathrm{C}$ ) and the off-transients were less than $1 \mathrm{mV}$ (7/8 flies, Figure 2D) in amplitude. Notably, additional light flashes did not evoke transients in older flies initially lacking transients. To extend our analysis of age-related changes in ERG waveforms, we also recorded ERGs across the lifespan of the longer-lived $23{ }^{\circ} \mathrm{C}$-reared population (Supplemental Figure 1). Similar to the observed trends in the $29{ }^{\circ} \mathrm{C}$-reared population, we found that the receptor potential amplitude was largely stable across the lifespan, and also noted a reduction in on- and off-transient amplitudes in the oldest flies compared to their younger counterparts. However, in most cases, both on- and off-transients still were detectable in aged individuals, consistent with previous observations (Phillips, Woodruff, Liang, Pattern, \& Broadie, 2008; Jaiswal et al., 2015). These findings suggest that over the course of normal-healthy aging, synaptic transmission between photoreceptors and $2^{\text {nd }}$ order targets in the lamina are selectively disrupted prior to any decline in the phototransduction cascade leading to the receptor potential.

\section{Two novel forms of ERG waveform oscillation}

During the course of our ERG experiments, we discovered that repetitive light flash protocols (flash duration: 50, or $500 \mathrm{~ms}$; inter-flash interval: 2.5, 0.5 or $0.1 \mathrm{~s}$; Figure 3A) led to two previously undocumented forms of oscillation in the ERG waveforms of young WT flies. The first form of oscillation appeared during ERG responses to 500-ms flashes. Although the initial ERG waveforms generally appeared to be normal, successive waveforms often displayed a distinctive oscillation during the off-transient (Figure 3B). These "light-off oscillations" had a 
1 frequency between 70 and $110 \mathrm{~Hz}$ and an amplitude that appeared to grow upon successive stimulations, suggesting an activity-dependent recruitment. Indeed, we found that these oscillations were most obvious, up to $1 \mathrm{mV}$ in amplitude, between the $8^{\text {th }}$ and $10^{\text {th }}$ light flash. Upon closer examination, however, several cases of subtle light-off oscillation were found in the off-transient of the first flash as well.

A second form of oscillation was observed during a sequence of short (50 ms) light flash trains (inter-flash interval: 2.5, 0.5 and $0.1 \mathrm{~s}$; Figure 3A). During these short flashes full ERG waveforms were not evoked; instead, only the on-transient and the initial portion of the RP were observed (Figure 3C). Successively evoked on-transients sometimes displayed a high frequency oscillation (between 100-200 Hz) immediately after the peak of the on-transient (Figure 3C).

11 These "light-on" oscillations were frequently observed between the $2^{\text {nd }}$ and $5^{\text {th }}$ light flash of the 10 flash train, and were approximately $0.3 \mathrm{mV}$ in size, smaller than the light-off oscillations. Light-on oscillations tended to appear in the later stimulus trains $(0.1 \mathrm{~s}$ inter-flash intervals, Figure 3A). However, the occurrence of light-on oscillations was not necessarily dependent on the inter-flash interval. When the stimulus protocol was reversed, the oscillations were observed during $2.5 \mathrm{~s}$ intervals, although the rate of observation was reduced. to eliminate a number of artefactual possibilities that could potentially drive the phenomena (data not shown): 1) The flash intensity oscillated. Using a linear phototransistor circuit (Panasonic AMS302) to measure light output, we noted that the flash intensity was roughly square, with a

21 slight $(\sim 5 \%)$ overshoot during the initial phase. The light output never oscillated during periods of observed ERG oscillation. 2) Oscillation was specific to a single electrophysiological rig. 
1 Laboratory class. Furthermore, using the same light flash protocols, ERG oscillations were observed using an electrophysiology rig in Dr. Chun-Fang Wu's laboratory. 3) ERG oscillations require a specific light source. Reducing light intensity by 50\% using a neutral density (ND) filters did not disrupt the ERG oscillations. Furthermore, oscillations could be triggered by a wide range of light wavelengths. Both a blue LED (wavelength: $470 \pm 20 \mathrm{~nm}$, Phillips Luxeon Rebel LED), as well as a white LED passed through a long-pass filter (Kodak Wratten \#12, cutoff wavelength $\sim 510 \mathrm{~nm}$ ) were able to trigger both types of oscillations. Indeed, in our experiences, across an assortment of "white" LEDs, all were able to trigger ERG oscillations in WT flies during repetitive light flashes.

Given the frequent observation of both light off- and light on-oscillations in the WT strain Canton-S, we wanted to determine whether these oscillations could be observed in other Drosophila WT strains. We examined ERG waveforms of two other commonly used laboratory D. melanogaster strains: Oregon-R and Berlin using the repetitive light flash protocol described above. Importantly, we observed both light off- and light on-oscillations were in these strains, and the frequencies of both types of oscillation were within the range seen in the Canton-S strain (Table 1). These findings suggest that the occurrence of either form of oscillation is likely not

17 due to a particular genetic background in D. melanogaster. We then extended our analysis of ERG oscillations to include WT strains of four additional Drosophila species euronotus,

19 funebris, americana, and hydei. As listed in Table 1, all four species displayed both kinds of

20 ERG oscillations. However, in several cases the observed oscillations varied in terms of

21 frequency compared to melanogaster (Table 1). Although additional work is required to

22 systematically characterize the mechanisms for this variation, it is clear from our initial results 
1 that ERG oscillations triggered by repetitive stimulation may be widely observed across Drosophila species.

Oscillations within ERG waveforms are a well-described phenotype of a number of

$6 \quad$ visual mutants (Kelly \& Suzuki, 1974; Wu \& Wong, 1977; Leung, Geng, \& Pak., 2000). Perhaps

7 the best characterized of these are mutants of the gene $\operatorname{ros} A$ (Wu \& Wong, 1977; independently

8 isolated as inebriated, Stern \& Ganetzky 1992) which encodes a putative $\mathrm{Na}^{+} / \mathrm{Cl}^{-}$-dependent

9 solute transporter (Soehenge et al., 1996; Burg et al., 1996) potentially involved in

10 neurotransmitter transport (Huang et al., 2002). We wanted to compare the ERG waveform

11 oscillations in $\operatorname{ros} A$ mutants with the light-on and light-off oscillations in WT flies described

12 here. Consistent with previous reports (Wu \& Wong, 1977; Gavin, Arruda, \& Dolph, 2007), our

13 ERG recordings of the allele $\operatorname{ros} A^{p 213}$ revealed that $500 \mathrm{~ms}$ light flashes did not evoke on- or off-

14 transients, but recruited a prominent oscillation during the RP phase of the waveform $(\sim 3 \mathrm{mV}$,

15 Figure 4A). The frequency of the RP oscillation varied somewhat between individuals (40 to 90

$16 \mathrm{~Hz}$ ), but ceased within $10 \mathrm{~ms}$ of the end of the light flash. Using the repetitive light flash

17 protocols previously described (Figure 3A), we found several differences in how light-off and

18 light-on oscillations were recruited in $\operatorname{ros} A$. Specifically, the repetitive $500 \mathrm{~ms}$ flash protocols

19 (inter-flash interval: 2.5 or $0.5 \mathrm{~s}$ ) were able to recruit strong light-off oscillations during the

20 repolarization phase of the waveform, often lasting tens of milliseconds (Figure 4A-B). Within

21 the same individual, the frequency of the light-off oscillations was lower than corresponding RP

22 oscillation frequency (Figure 4B). Compared to WT counterparts, the light-off oscillations in

$23 \operatorname{ros} A$ were significantly larger in amplitude $(\sim 3 \mathrm{mV}$ vs $<1 \mathrm{mV})$ and generally had a lower 
1 oscillation frequency $(\sim 50 \mathrm{~Hz}$, Figure 4B). In contrast to the enhanced light-off oscillations in $\operatorname{ros} A$, we found that the $50 \mathrm{~ms}$ light flash protocols could not recruit light-on oscillations in $\operatorname{ros} A$. Taken together, these results seem to indicate a critical role for the rosA gene product in shaping

4 light-off and light-on oscillations in addition to suppressing RP oscillations in WT ERG waveforms.

\section{Age-dependent suppression of ERG waveform oscillations}

Although repetitive light flashes evoke light-off oscillations reliably and often trigger light-on oscillations in young flies, we noticed that these oscillations were largely absent from

9 the older WT flies we examined. As shown in Figure 5A, in $29^{\circ} \mathrm{C}$-reared WT flies, we observed

10 light-off oscillations in 23 out of 24 flies less than $15 \mathrm{~d}$ old (corresponding to $~ 50 \%$ of the

11 median lifespan), while in older flies, only 3 out of 11 displayed oscillation. Interestingly, the

12 frequency of light-off oscillation was also age dependent, with relatively younger flies displaying

13 higher frequency oscillations compared to older counterparts (Spearman's rank correlation test, $p$

$14<0.05)$. The proportion of flies displaying light-on oscillations also decreased with age: 10 out of

1523 flies for flies less than $15 \mathrm{~d}$ old vs. 1 out of 9 for flies older than $15 \mathrm{~d}$. However, we did not

16 detect a significant age dependence in the light-on oscillation frequency (Figure 5B). Based on

17 these observations, loss of light-on or light-off transients may serve as a marker of age-

18 dependent alterations in the Drosophila visual system alongside the attenuation of ERG

19 transients. 


\section{Discussion}

Our study compares the progression of age-related changes in phototactic behavior with changes in the Drosophila ERG waveform. We observed an age-related decline in phototaxis during which negative geotaxis within the same population was consistently stronger (Figure 1). The relative strength of negative geotaxis suggests that the decline in phototaxis may be due to disruption of requisite sensory inputs rather than motor system function. Indeed, previous findings using different methodologies have shown a similar decline in phototaxis which precedes a loss of motor coordination in aging flies (Leffelaar \& Grigliatti, 1983; Arking \& Wells, 1990). Interestingly, we noted that the decline in phototaxis was paralleled with the agerelated decrease of on- and off-transients in ERG waveforms (Figure 2). On- and off-transients serve to indicate integrity of synaptic transmission between photoreceptors and $2^{\text {nd }}$ order targets (Coombe, 1986), and disruption of transients leads to distinct phototaxis deficits (Pak, 1975).

These results suggest that the loss of ERG transients may contribute to the observed loss of phototaxis. Among the $23{ }^{\circ} \mathrm{C}$-reared WT population aged beyond $40 \mathrm{~d}$, we found that flies with a phototaxis score of ' 3 ' had larger average off-transient amplitudes than those with a score of ' 0 '. However, a limited sample size of old flies displaying good phototaxis precluded robust statistical analysis, and we identified a few individuals with a 'poor' phototaxis score which displayed robust transients. Further study, perhaps utilizing a behavioral assay sensitive to individuals such as the opto-motor response in a tethered flight simulator (Götz, 1968; Götz, Hengstenberg, \& Biesinger, 1979) may more directly correlate the loss of transients with visually mediated behaviors within individual aged flies.

Notably, a loss of ERG transients coupled with a decline in visually mediated behaviors is a phenotype associated with a number of neurodegeneration mutants (e.g. optomotor blind, 
1 Heisenberg, Wonneberger, \& Wolf, 1978; swisscheese, Kretzschmar, Hasan, Sharma, Heisenberg, \& Benzer, 1997) which also display clear disruptions in photoreceptor or laminar structure (Pflugfelder et al., 1990; Pflugfelder et al., 1992; Kretzschmar, et al., 1997; Kretzschmar 2009). It is possible that similar structural defects in photoreceptor, laminar and/or surrounding glial cells may contribute the observed decline in transients in aged flies. Furthermore, direct monitoring of the post-synaptic potentials in laminar and/or medullar cells perhaps via direct patch recordings (Skingsley, Laughlin, \& Hardie, 1995; Tuthill, Nern, Rubin, \& Reiser, 2014) or $\mathrm{Ca}^{2+}$ imaging (Clark, Bursztyn, Horowitz, Schnitzer, \& Clandinin, 2011) would be potentially useful in pinpointing the physiological basis for this decline. Previous work in other systems have shown age dependent changes in field recordings of synaptic transmission (Koss, Drever, Stoppelkamp, Riedel, \& Platt, 2013; Piskorowski et al., 2016) and circuit activity (Hughes \& Cayaffa, 1977; Landholt \& Borbely, 2001). 
Although oscillations can be observed in the electrical activity of isolated mutant photoreceptor cells with the use of intracellular recordings (Wu \& Wong, 1977), the ERG waveform oscillations likely reflect collectively synchronous activity of many photoreceptor cells and their synaptic connections. The relative resistance between photoreceptor cells is much smaller than the resistance across the basement membrane. Thus, when the probe electrode and the ground electrode are placed on opposite sides of the retina's basement membrane, the resulting ERG waveform represents the combined activity of photoreceptors and their synaptic targets (Heisenberg, 1971; Stark \& Wasserman, 1974). Oscillations may arise through an interaction between photoreceptor activities, such as feedback amplification, which serves to synchronize them. A glial cell syncytium connected through gap junctions (Stebbings et al., 2002; Chaturvedi, Reddig, \& Li, 2014) could contribute an important mechanism to synchronize photoreceptor and/or laminar activity, facilitating the reported ERG oscillations.

The two separate ERG oscillations identified may involve distinct combinations of physiological mechanisms involving photoreceptor cells, postsynaptic monopolar laminar cells, as well as surrounding glial cells (Heisenberg, 1971; Coombe, 1986; Xiong \& Montell, 1995).

The different stimulus recruitment protocols and oscillation frequencies of light-on and light-off oscillations (Figure 3, Figure 5) suggest that they arise through independent mechanisms. Indeed, we noted several WT individuals which displayed light-off but not light-on oscillations (Table 1). As mentioned above, light-on oscillations were absent in flies with attenuated on-transients including both $\operatorname{ros} A$ mutants and older WT flies, suggesting that light-on oscillations require intact on-transients. (Preliminary analysis of the transient-less mutant lines $h d c^{p 211}$ and $o r t^{j k 84}$ also indicates a loss of light-on oscillations; I.M., unpublished observations.) Light-off oscillations, in contrast, do not require intact off-transients, as rosA mutants which lacked off- 
1 transients displayed strong light-off oscillations in response to repetitive light flashes (Figure 4).

2 However, our observations indicate a potential role for the $\operatorname{ros} A$ gene product in the light-off

3 oscillations. In $\operatorname{ros} A$ mutants, the light-off oscillations are distinct from previously described RP

4 oscillations in terms of frequency and location within the ERG waveform (Figure 4).

5 Furthermore, the frequency of light-off oscillations in $\operatorname{ros} A$ is reduced and the amplitude is

6 increased compared to WT light-off oscillations. Although the precise physiological mechanisms

7 by which $\operatorname{ros} A$ mutations induce RP oscillations remain unclear (Gavin et al., 2007), the $\operatorname{ros} A$

8 gene product along with other neurotransmitter transporters (e.g. carT, see Xu et al., 2015;

9 Chaturvedi et al., 2016) may play an important role in shuttling histamine and related

10 metabolites across the plasma membranes of photoreceptors and surrounding glia in the visual

11 system - a potentially important mechanism in generating ERG waveform oscillations. 


\section{References}

Alawi, A., \& Pak, W. (1971). On-transient of insect electroretinogram: its cellular origin. Science, 172, 1055-1057.

Arking, R., \& Wells, R. (1990). Genetic alteration of normal aging processes is responsible for extended longevity in Drosophila. Genesis, 11, 141-148.

Atwood, H. (1992). Age-dependent alterations of synaptic performance and plasticity in crustacean motor systems. Exp. Geronotol., 27, 51-61.

Benzer, S. (1967). Behavioral mutants of Drosophila isolated by countercurrent distribution. Proc. Natl. Acad. Sci. USA, 58, 1112-1119.

Bloomquist, B., Shortridge, R., Schneuwly, S., Perdew, M., Montell, C., Steller, H., . . Pak, W. (1988). Isolation of a putative phospholipase c gene of Drosophila, norpA, and its role in phototransduction. Cell, 54, 723-733.

Burg, M., Geng, C., Guan, Y., Koliantz, G., \& Pak, W. (1996). Drosophila rosA gene, which when mutant causes aberrant photoreceptor oscillation, encodes a novel neurotransmitter transporter homologue. J. Neurogenet., 11, 59-79.

Chaturvedi, R., Reddig, K., \& Li, H.-S. (2014). Long-distance mechanism of neurotransmitter recycling mediated by glial network facilitates visual function in Drosophila. Proc. Natl. Acad. Sci. USA, 111(7), 2812-2817.

Chaturvedi, R., Luan, Z., Guo, P., \& Li, H.-S. (2016) Drosophila vision depends on carcinine uptake by an organic cation transporter. Cell. Rep., 14, 2076-2083.

Chouhan, A., Guo, C., Hsieh, Y.-C., Ye, H., Senturk, M., Zuo, Z., . . Shulman, J. (2016). Uncoupling neuronal death and dysfunction in Drosophila models of neurodegenerative disease. Acta Neuropathologica Comm., 4.

Clapham, D., Runnels, L., \& Strubing, C. (2001). The TRP ion channel family. Nat. Rev. Neurosci., 2, 387-396.

Clark, D., Bursztyn, L., Horowitz, M., Schnitzer, M., \& Clandinin, T. (2011). Defining the computational structure of the motion detector in Drosophila. Neuron, 70, 1165-1177.

Coombe, P. (1986). The large monopolar cells L1 and L2 are responsible for ERG transients in Drosophila. J. Comp. Physiol. A, 159, 655-665.

Dolph, P., Nair, A., \& Raghu, P. (2010). Analysis of visual physiology in the adult Drosophila eye. In B. Zhang, M. Freeman, \& S. Waddell (Eds.), Drosophila Neurobiology: A Laboratory Manual (pp. 227-235). Cold Spring Harbor, NY: Cold Spring Harbor Press.

Frankel, A., \& Brosseau, G. (1968). A Drosophila medium that does not require dried yeast. Drosophila Information Service, 43, 184. 
Gavin, B., Arruda, S., \& Dolph, P. (2007). The role of carcinine in signalling at the Drosophila photoreceptor synapse. PLOS Genet., 3(12), e206.

Govind, C. (1992). Age-related remodeling of lobster neuromuscular terminals. Exp. Geronotol., 27(1), 63-74.

Götz, K. (1968). Flight control in Drosophila by visual perception of motion. Kybernetik, 4, 199208.

Götz, K., Hengstenberg, B., \& Biesinger, R. (1979). Optomotor control of wing beat and body posture in Drosophila. Biol. Cybernet., 35, 101-112.

Heisenberg, M. (1971). Separation of receptor and lamina potentials in the electroretinogram of normal and mutant Drosophila. J. Exp. Biol., 55, 85-100.

Heisenberg, M. \& Wolf, R. (1984). Vision in Drosophila: Genetics of microbehavior. Berlin Heidelberg: Springer-Verlag.

Heisenberg, M., Wonneberger, R., \& Wolf, R. (1978). Optomotor-blind H31-a Drosophila mutant of the lobula plate giant neurons. J. Comp. Physiol. A, 1978, 287-296.

Huang, Y., \& Stern, M. (2002). In vivo properties of the Drosophila inebriated-encoded neurotransmitter transporter. J. Neurosci., 22, 1698-1708.

Hughes, J., \& Cayaffa, J. (1977). The EEG in patients at different ages without organic cerebral disease. Electroencephalography and Clin. Neurophysiol., 42, 776-784.

Jaiswal, M., Haelterman, N., Sandoval, H., Xiong, B., Donti, T., Kalsotra, A., ... Bellen, H. (2015). Impaired mitochondrial energy production causes light-induced photoreceptor degeneration independent of oxidative stress. PLOS Biol., 13, e1002197.

Kelly, L., \& Suzuki, D. (1974). The effects of increased temperature on electroretinograms of temperature-sensitive paralysis mutants of Drosophila melanogaster. Proc. Natl. Acad. Sci. USA, 71, 4906-4909.

Koss, D., Drever, B., Stoppelkamp, S., Riedel, G., \& Platt, B. (2013). Age-dependent changes in hippocampal synaptic transmission in the PLB1_triple Alzheimer mouse. Cell. Mol. Life Sci., 70, 2585-2601.

Kretzschmar, D. (2009). Swiss cheese et allii, some of the first neurodegenerative mutants isolated in Drosophila. J. Neurogenet., 23, 34-41.

Kretzschmar, D., Hasan, G., Sharma, S., Heisenberg, M., \& Benzer, S. (1997). The swisscheese mutant causes glial hyperwrapping and brain degeneration in Drosophila. J. Neurosci. 17, 7425-7432.

Landholt, H.-P., \& Borbely, A. (2001). Age-dependent changes in sleep EEG topography. Clin. Neurophysiol., 112, 369-377. 
Lee, W.-C., Yoshihara, M., \& Littleton, J. (2003). Cytoplasmic aggregates trap polyglutaminecontaining proteins and block axonal transport in a Drosophila model of Huntington's Disease. Proc. Natl. Acad. Sci. USA, 101, 3224-3229.

Leffelaar, D., \& Grigliatti, T. (1983). Age-dependent behavior loss in adult Drosophila melanogaster. Genesis, 4, 211-227.

Leung, H.-T., Geng, C., \& Pak, W. (2000). Phenotypes of trpl mutants and interactions between the transient receptor potential (TRP) and TRP-like channels in Drosophila. J. Neurosci., 20, 6797-6803.

Martinez, V., Javadi, C., Ngo, E., Ngo, L., Lagow, R., \& Zhang, B. (2007). Age-related changes in climbing behavior and neural circuit physiology. Dev. Neurobiol., 67, 778-791.

Minke, B., Wu, C.-F., \& Pak, W. (1975). Induction of photoreceptor voltage noise in the dark in Drosophila mutant. Nature, 258, 84-87.

Montell, C., \& Rubin, G. (1989). Molecular characterization of the Drosophila trp locus: a putative integral membrane protein required for phototransduction. Neuron, 2, 13131323.

Oortveld, M., Keerthikumar, S., Oti, M., Nijhof, B., Fernandes, A., Kochinke, K., . . Schenck, A. (2013). Human intellectual disability genes form conserved functional modules in Drosophila. PLOS Genetics, 9(10), e1003911.

Pak, W. (1975). Mutations affecting the vision of Drosophila melanogaster. In R. King (Ed.), Handbook of Genetics (Vol. 3, pp. 703-733). New York, NY: Plenum Publishing.

Pak, W. (2010). Why Drosophila to study phototransduction? J. Neurogenet., 24, 55-66.

Pak, W., Grossfield, J., \& White, N. (1969). Nonphototactic mutants in a study of vision of Drosophila. Nature, 222, 351-354.

Pflugfelder, G., Roth, H., Poeck, B., Kerscher, S., Schwarz, H., Jonschker, B., \& Heisenberg, M., (1992). The lethal (1) optomotor-blind gene of Drosophila melanogaster is a major organizer of optic lobe development: isolation and characterization of the gene. Proc. Natl. Acad. Sci. USA, 89, 1199-1203.

Pflugfelder, G., Schwarz, H., Roth, H., Poeck, B., Sigl, A., Kerscher, S., ... Heisenberg, M. (1990). Genetic and molecular characterization of the optomotor-blind gene locus in Drosophila melanogaster. Genetics, 126, 91-104.

Phillips, S., Woodruff, E., Liang, P., Pattern, M., \& Broadie, K. (2008). Neuronal loss of Drosophila NPC1a causes cholesterol aggregation and age-progressive neurodegeneration. J. Neurosci., 28, 6569-6582.

Piskorowski, R., Nasrallah, K., Diamantopoulou, A., Mukai, J., Hassan, S., Siegelbaum, S. G., \& Chevaleyre, V. (2016). Age-dependent specific changes in area CA2 of the hippocampus 
and social memory deficit in a mous model of the 22q11.2 deletion syndrome. Neuron, 89, 163-176.

Rahman, M., Ham, H., Liu, X., Sugiura, Y., Orth, K., \& Kramer, H. (2012). Visual neurotransmission in Drosophila requires expression of Fic in glial capitate projections. Nat. Neurosci., 15, 871-875.

Ruan, H. (2008). On Drosophila aging: Lifespan plasticity, social-behavioral influences and neurophysiological indices. (PhD Thesis). University of Iowa, Iowa City, IA, USA.

Ruan, H., \& Wu, C.-F. (2008). Social interaction-mediated lifespan extension of Drosophila Cu/Zn superoxide dismutase mutants. Proc. Natl. Acad. Sci. USA, 105, 7506-7510.

Skingsley, D., Laughlin, S., \& Hardie, R. (1995). Properties of histamine-activated chloride channels in the large monopolar cells of the Dipteran compound eye: a comparative study. J. Comp. Physiol. A, 176, 611-623.

Soehenge, H., Huang, X., Becker, M., Whitley, P., Conover, D., \& Stern, M. (1996). A neurotransmitter transporter encoded by the Drosophila inebriated gene. Proc. Natl. Acad Sci. USA, 93, 13262-13267.

Stark, W., \& Wasserman, G. (1974). Wavelength-specific ERG characteristics of pigmented and white-eyed strains of Drosophila. J. Comp. Physiol. A, 91, 427-411.

Stebbings, L., Todman, M., Phillips, R., Greer, C., Tam, J., Phelan, P., ... Davies, J. (2002). Gap junctions in Drosophila: developmental expression of the entire innexin gene family. Mech. Dev., 113, 197-205.

Stern, M., \& Ganetzky, B. (1992). Identification and characterization of inebriated, a gene affecting neuronal excitability in Drosophila. J. Neurogenet., 8, 157-172.

Tuthill, J., Nern, A., Rubin, G., \& Reiser, M. (2014). Wide-field feedback neurons dynamically tune early visual processing. Neuron, 82, 887-895.

Vilinsky, I., \& Johnson, K. (2012). Electroretinograms in Drosophila: A robust and genetically accessible electrophysiological system for the undergraduate laboratory. J. Undergrad. Neurosci. Educ., 11, A149-A157.

West, R., Elliott, C., \& Wade, A. (2015). Classification of Parkinson's Disease genotypes in Drosophila. Sci. Rep., 5, 16933.

Wu, C.-F., \& Wong, F. (1977). Frequency characteristics in the visual system of Drosophila. Genetic dissection of electroretinogram components. J. Gen. Physiol., 69, 705-724.

Xiong, W.-C., \& Montell, C. (1995). Defective glia induce neuronal apoptosis in the repo visual system of Drosophila. Neuron, 14, 581-590. 
1 Xu, Y., An, F., Borycz, J., Borycz, J., Meinertzhagen, I., \& Wang, T. (2015). Histamine

2

3

4

5

15

16 recycling is mediated by CarT, a carcinine transporter in Drosophila photoreceptors. PLOS Genet., 11, e1005764.
Yamamoto, S., Jaiswal, M., Charng, W.-L., Gambin, T., Karaca, E., Mirzaa, G., . . Bellen, H. (2014). A Drosophila genetic resource of mutants to study mechanisms underlying human genetic diseases. Cell, 159, 200-214.

8 
bioRxiv preprint doi: https://doi.org/10.1101/259952; this version posted February 5, 2018. The copyright holder for this preprint (which was not certified by peer review) is the author/funder, who has granted bioRxiv a license to display the preprint in perpetuity. It is made available under aCC-BY-NC-ND 4.0 International license.

\section{Table 1}

2

\begin{tabular}{|r|c|c|c|c|}
\hline \multirow{2}{*}{ Species (Strain) } & \multicolumn{2}{|c|}{ Light-off oscillation } & \multicolumn{2}{c|}{ Light-on oscillation } \\
\cline { 2 - 5 } & Fraction & Freq. Range (Hz) & Fraction & Freq. Range (Hz) \\
\hline D. melanogaster (Canton-S) & $19 / 20$ & $70-110$ & $9 / 19$ & $100-200$ \\
\hline D. melanogaster (Oregon- ) & $4 / 4$ & $80-100$ & $2 / 4$ & $\sim 140$ \\
\hline D. melanogaster (Berlin) & $5 / 5$ & $70-100$ & $3 / 5$ & $100-150$ \\
\hline D. euronotus & $4 / 5$ & $60-100$ & $5 / 5$ & $50-150^{*}$ \\
\hline D. americana & $5 / 5$ & $70-150$ & $5 / 5$ & $60-150^{*}$ \\
\hline D. hydei & $5 / 5$ & $80-120$ & $5 / 5$ & $100-120$ \\
\hline D. funebris & $4 / 5$ & $80-120$ & $5 / 5$ & $60-120$ \\
\hline
\end{tabular}

* Substantial frequency variation within individuals 


\section{Figure Legends}

2 Figure 1. Distinct progression of age-dependent decline in phototaxis and negative geotaxis. (A) $29{ }^{\circ} \mathrm{C}$-reared individuals. (B) $23{ }^{\circ} \mathrm{C}$-reared individuals. Points represent the mean photo/geo-taxis index for WT flies. Error bars indicate SEM. ${ }^{*} p<0.05$, *** $p<0.001$, KruskalWallis ANOVA, Bonferroni-corrected rank-sum post hoc test. $\mathrm{n}=7$ - 40 flies per run, 2 - 4 runs per age, number of total flies per age group as indicated in parenthesis)

Figure 2 Age-dependent alterations of ERG waveforms in $29^{\circ} \mathrm{C}$-reared WT flies.

(A) Representative ERGs from 8 and 30 d flies triggered by a $500 \mathrm{~ms}$ light flash. ERG as depicted in the $8 \mathrm{~d}$ sample ERG. Note that on- and off-transients are attenuated in the $30 \mathrm{~d}$ example. (B-D) Age-dependent alterations in the amplitudes of (B) RP, (C) on-transient, and (D) off-transients. Trend-lines indicate a Gaussian-kernel running average ( $\sigma=5 \mathrm{~d}) . \mathrm{n}=42$ flies.

Figure 3. ERG oscillations triggered by repetitive light flashes. set of three trains used $500 \mathrm{~ms}$ flashes delivered at 2.5, 0.5 and $0.1 \mathrm{~s}$ intervals, while the second set used $50 \mathrm{~ms}$ flashes delivered in the same manner. (B) Two representative ERG traces evoked by $500 \mathrm{~ms}$ flashes. The off-transients are enlarged to the right. The lower trace displays light-off

21 flashes (lower panel). The on-transients are enlarged to the right. The trace above shows light-on oscillations (arrows), while the trace below does not. 
1 Figure 4. Light-off oscillations in the visual system mutant $\operatorname{ros} A$.

2 (A) ERG waveforms evoked by a $500 \mathrm{~ms}$ light flash in WT and $\operatorname{ros} A$ mutants. Upper traces: $1^{\text {st }}$

3 flash, lower traces: $6^{\text {th }}$ flash; inter-flash interval: 500 ms. Note the oscillations during the RP and

4 absence of transients in $\operatorname{ros} A$. (B) Expansion of the off-transient and repolarization. Arrows

5 indicate light-off oscillations, asterisks indicate RP oscillations in $\operatorname{ros} A$, and circles indicate light-

6 off artifact.

Figure 5. Age-related changes in light-off and light-on oscillations

9 (A) Scatterplot of age versus oscillation frequency for light-off oscillations in WT flies and (B)

10 light-on oscillations. Sample sizes as indicated in upper-right corner. Note that flies that did not

11 display oscillations are indicated at the bottom of each plot. A Fisher's exact test revealed that

12 both light-on and light-off oscillations were more frequently observed in younger flies $(p<0.001$

13 for light-off oscillation, $p<0.05$ for light-on oscillation).

15 Supplemental Figure 1 Age-dependent alterations of $23{ }^{\circ} \mathrm{C}$-reared WT ERG waveforms.

16 The amplitudes of (A) RP, (B) on-transient, and (C) off-transient. Trend-lines indicate a

17 Gaussian-kernel running average $(\sigma=5 \mathrm{~d}) . \mathrm{n}=19$ flies. 


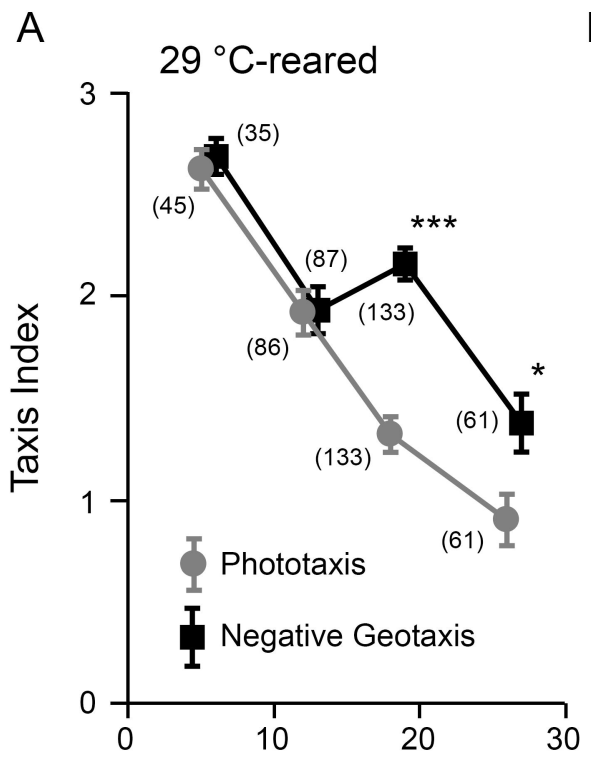

B

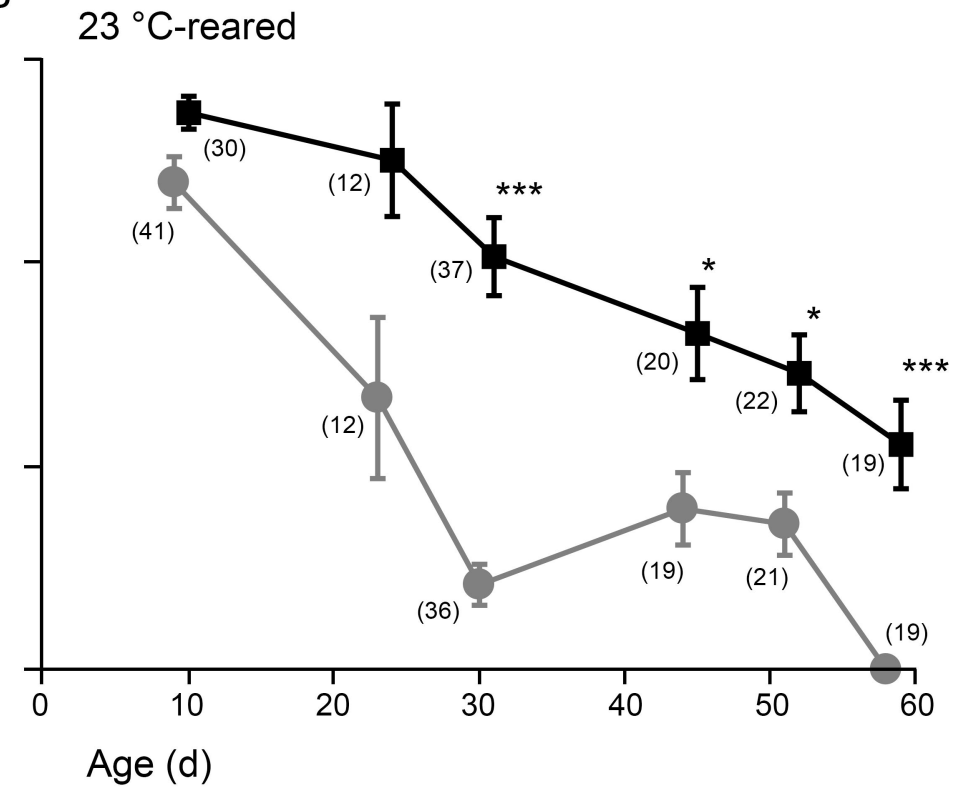


A WT $29^{\circ} \mathrm{C}$-reared

$8 \mathrm{~d}$

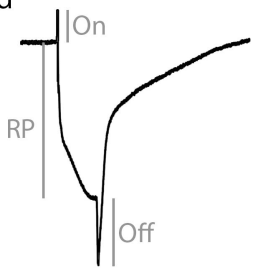

$30 \mathrm{~d}$

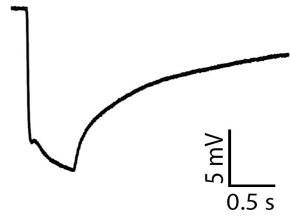

B

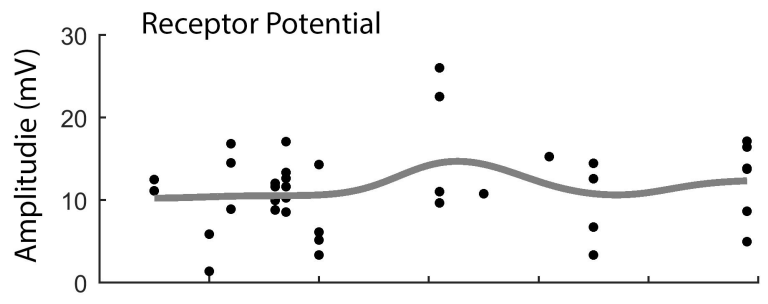

C

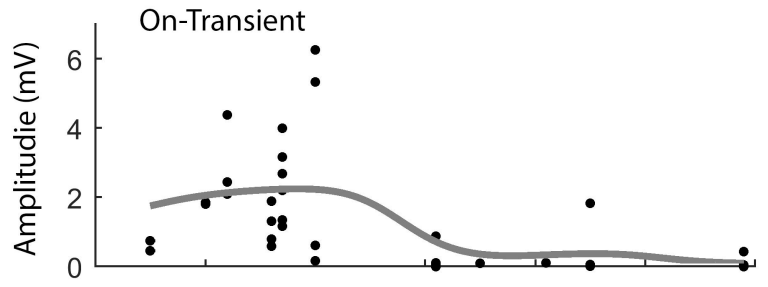

D

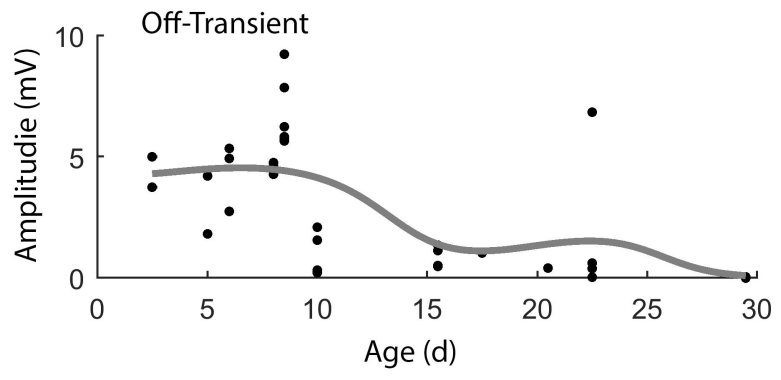


$A$

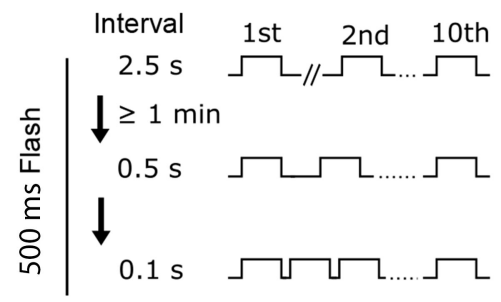

$\downarrow$

$\frac{c}{4}$
B flight-Off Oscilations

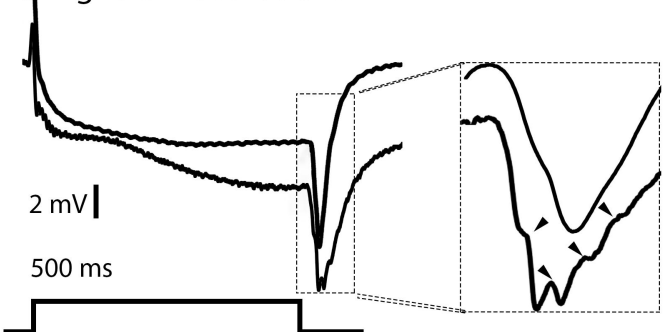

C

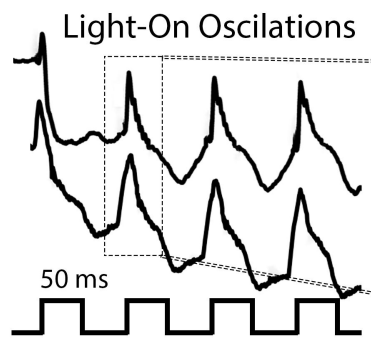

$1 \mathrm{mv}$ $30 \mathrm{~ms}$ 

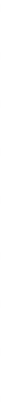

B

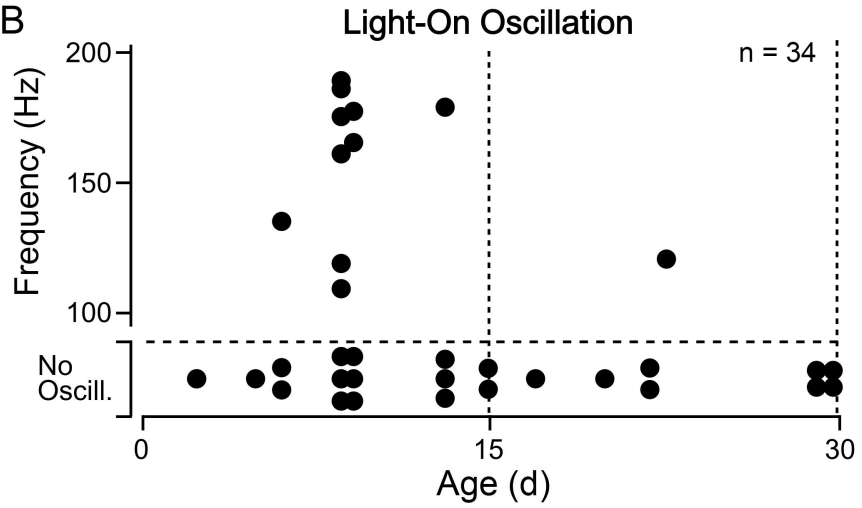

Light-On Oscillation

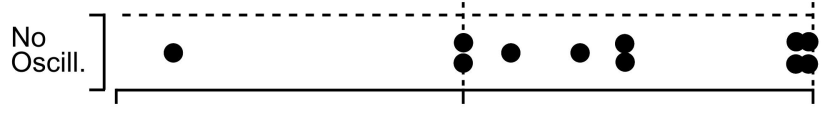

